\title{
A Continuously Adaptive MLSE Receiver for Mobile Communications: Algorithm and Performance
}

\author{
Guido Castellini, Fabrizio Conti, Enrico Del Re, Senior Member, IEEE, and Laura Pierucci
}

\begin{abstract}
The paper presents a Euclidean distance maximum likelihood sequence estimation (MLSE) receiver, based on the Viterbi algorithm (VA), suitable for fading and noisy communications channels, as that specified by the group spécial mobiles (GSM). In a mobile cellular system, the fast varying channel characteristics, due to the fading and Doppler effects, require adaptive methods to update the channel coefficients to the MLSE receiver. The proposed technique continuously estimates the channel characteristics directly within the metric calculation of the VA. At each step of the VA, the sequence associated to the path with the best metric value (minimum-survivor method) among the survivor paths is used to update the channel estimate (employing conventional adaptive algorithms) throughout the entire informative sequence. However, the detection of the transmitted data sequence is performed by the VA only at the end of each burst.

The proposed technique allows simpler receiver implementation and the simulation results show a good performance of this adaptive MLSE receiver in typical GSM environments.
\end{abstract}

Index Terms - Adaptive algorithms, fading channels, MLSE receiver, Viterbi algorithm.

\section{INTRODUCTION}

$\mathbf{T}$ HE digital land mobile radio system as specified by the European Telecommunications Standards Institute/Group Spécial Mobiles (ETSI/GSM) has been put into service in several European countries. The narrow band time-division multiple-access (TDMA) digital cellular system has required the channel coding, interleaving and compact-spectrum constant envelope modulation [the Gaussian minimum shift keying, (GMSK)] to increase the spectrum efficiency. Due to the spectral shape of the adopted partial response modulation and to the multipath nature of the communication channel, interference occurs between adjacent symbols, which are known as inter-symbol interference (ISI). The best theoretical performance for demodulating operations over channels with ISI and additive white noise is the maximum likelihood sequence estimation (MLSE) technique [3]-[6] which is implemented by means of the Viterbi algorithm (VA). On the additive

Paper approved by N. C. Beaulieu, the Editor for Wireless Communication Theory of the IEEE Communications Society. Manuscript received August 15, 1994; revised January 12, 1995. This paper was supported by MURST and the National Research Council Special Project.

G. Castellini is with the Institute of Research on Electromagnetic Waves (IROE) of the Italian National Research Council (CNR), Florence 50127 Italy (email: guido@alpha1.iroe.fi.cnr.it).

F. Conti is with the Alcatel Siette Division of Alcatel Italia S.p.A., Sesto Fiorentino, 50019 Italy (email: Fabrizio.Conti@sie.alcatel.it).

E. Del Re and L. Pierucci are with the Department of Electronics Engineering, University of Florence, Florence 50139 Italy (email: delre@cosimo.die.unifi.it and pierucci@cosimo.die.unifi.it).

Publisher Item Identifier S 0090-6778(97)00729-0. white Gaussian noise (AWGN) channel, the MLSE criterion is equivalent to a receiver which minimizes the Euclidean distance between the received sequence and all the possible transmitted sequences.

This Euclidean MLSE receiver is analyzed in the paper since no matched filter is needed and, in view of adaptive implementations, the Viterbi processor directly gives the error signal used by the adaptive channel estimation algorithms. The GSM communication protocol establishes reference data sequences in the data packet to determine the channel characteristic: however, the mobile environment, the TDMA signal structure and the rapidly varying channel characteristics, due to fading and Doppler effects, require adaptive techniques through a continuous updating of channel characteristics. The conventional adaptive MLSE receiver [3], [5] has difficulty in tracking fast time-varying ISI channels due to the fixed decision delay inherent in the VA which causes a channel estimation delay. In the literature [7], [28], the per-survivor processing (PPSP) and the adaptive MLSE proposed by Kubo et al. are presented, which utilize data-aided estimation techniques within the VA without being influenced by the fixed decision delay. In these methods, the estimate of the channel impulse response is evaluated using the data sequence associated to each survivor path in the branch metric calculation, which is relative to a possible state transition in the Viterbi trellis. Therefore, for each survivor, independent channel coefficients are updated employing conventional adaptive algorithms.

The paper presents a method in which the parameters update is carried out with a restricted delay recursively at each step of the VA during the unknown information sequence, taking into account the data sequence that corresponds to the best metric value to be the reference one (minimum-survivor method) [5], [18], [19]. The selected data sequence is considered to be one of the closest to the received signal up to that time, and is used to update the channel estimate of all survivors. However, the decision on the transmitted data sequence is taken by the VA at the end of each burst. Our investigation is similar to PPSP, but in the final analysis the min-survivor method highlights a significantly lower computational complexity with respect to PPSP, giving equivalent performance in typical GSM environments as shown in the simulation results.

The paper is organized as follows. Section II describes the linear model for the GMSK modulation as belonging to the class of the CPM modulation and gives the model of the used communication channel, including fading and Doppler effects. In Section III the structure of the proposed MLSE receiver is analyzed and the classical adaptive algorithms, such 
as the least mean square (LMS) and the recursive least square (RLS) algorithms used within the VA (min-survivor and persurvivor methods), are described. Finally, in Section IV the performance of the adaptive MLSE receivers in terms of biterror rate (BER) versus the ratio energy per bit to noise spectral density $\left(E_{b} / N_{0}\right)$ is shown, and the conclusions can be found in Section V.

\section{LINEAR MODEL OF CPM SIGNALS WITH $h=1 / 2$ AND ChanNEL EFFECTS ON SignAL}

In this section, we briefly recall the relevant characteristics of continuous phase modulation (CPM) signals with a modulation index $h=1 / 2$ and the model of the communication channel used. Any CPM signal can be defined by the continuous phase shift function $\varphi(t)$. In terms of complex envelope representation, it has the general form [1]

$$
r_{0}(t)=e^{j \pi h \sum_{n} x_{n} \varphi(t-n T)}
$$

where $T$ is the bit period, $x_{n}= \pm 1$ is the sequence of binary alphabet symbols and $j=\sqrt{ }-1$. The phase shift function is assumed to be zero for negative values of time $t$ and to have constant value for $t$ greater than $\left(L_{m}-1\right) T, L_{m}$ being a positive integer. Examples of modulations belonging to this class are minimum shift keying (MSK) and GMSK [1], [8]. As shown [2], the $h=1 / 2 \mathrm{CPM}$ signal can be very closely approximated by a sum of time and phase shifted pulses as follows:

$$
r_{1}(t)=\sum_{n} j^{n} z_{n} q(t-n T) \simeq r_{0}(t)
$$

In (2) the $z_{n}$ symbols are determined by the recursion

$$
z_{n}=x_{n} z_{n-1}
$$

and the pulse $q(t)$, which approximates the form of the modulation pulse shape and has a duration of less than or equal to $L_{m} T$, is defined by

$$
q(t)=q_{0}(t)=\prod_{i=0}^{L_{m}-2} \sin \left[\frac{\pi}{2} \psi(t+i T)\right]
$$

where

$$
\psi(t)= \begin{cases}\varphi(t) & t \leq\left(L_{m}-1\right) T \\ 1-\varphi\left(t-\left(L_{m}-1\right) T\right) & t>\left(L_{m}-1\right) T\end{cases}
$$

The factor $j^{n}$ in (2), which causes $\pi / 2$ phase rotation on the complex plane from symbol to symbol, can be avoided by means of a derotation technique [9], e.g., by multiplying, at the receiver, the signal $r_{1}(t)$ by the complex function: $d(t)=(-j)^{i}$ for $i T \leq t<(i+1) T$.

Hence, the signal takes the form

$$
r_{2}(t)=d(t) r_{1}(t)=\sum_{n} z_{n} p(t-n T)
$$

having defined a derotated pulse $p(t)=d(t) q(t)$.

Finally, let $a_{n}$ be the assigned binary sequence to be modulated. In order to have $a_{n}$ in the linear representation (6), the transmitter should build the phase in the exponent of (1) after a differential encoding of the sequence: from (3), choosing $x_{n}=a_{n} a_{n-1}$, it results $\left\{z_{n}\right\}=\left\{a_{n}\right\}$.

In this way, an $h=1 / 2$ CPM modulation can be depicted as a simple binary pulse amplitude modulation (PAM) signal, thus simplifying the signal model and the receiver structure. In the following, the linear model

$$
r(t)=\sum_{n} a_{n} p(t-n T)
$$

will be assumed for the baseband transmitted signal.

The parameter $L_{m}$ represents the duration (in symbols) of the modulating pulse $p(t)$ and, consequently, of the controlled intersymbol interference (ISI) inherent to the modulation itself. Typical values of $L_{m}$ are in the range two to four.

In land mobile radio, the received signal is subjected to multipath components caused by the reflections from a large number of objects in the surroundings of the mobile antenna. These signal components are received with independently time-varying amplitudes and phases, with random incoming angle and time delay [10]. According to the GSM recommendations [11], [12], the channel can be regarded as a time-variant linear system where each path is characterized by a delay $d_{i}(t)$ and by a specific attenuation $A_{i}(t)$.

The adopted channel model is composed of six to twelve distinct propagation paths which are subject to Rayleigh fading. For all Rayleigh fading paths, we adopt the classical Doppler spectrum as derived in [11]-[13]. The baseband expression for the noiseless received signal is

$$
s(t)=\sum_{n} a_{n} c(t, t-n T)
$$

where

$$
c(t, \tau)=\int p(\bar{t}) h(t, \tau-\bar{t}) d \bar{t}
$$

In (9), $h(t, \tau)$ represents the lowpass impulse response of the channel and $c(t, \tau)$ is the overall equivalent impulse response of the communication channel, including the effects of transmitter and receiver bandpass filters on the pulse shape. The time variation of the impulse response depends on the vehicle speed, the wavelength of the carrier and the scattering process. In the applications of interest, the channel model can be considered constant during a time interval equal to at least some bit periods, since the bit rate $f_{t}=1 / T$ is much greater than the Doppler frequency even for high vehicle speed. The dependence of the impulse response from variable $\tau$ determines instead the shape of the received pulse. Let $L_{C}$ be the duration of $h(t, \tau)$ expressed in units of $T$. Then the duration of the ISI in the received signal exceeds the quantity $L_{m}$, intrinsic in the modulation itself, up to $L_{S}=L_{m}+L_{C}$. In the system under examination, typical values for $L_{C}$ parameter are one through two in rural or urban environments [12]. Let $n(t)$ be the baseband additive noise introduced in the channel, in such a way that the baseband received signal expression is

$$
y(t)=s(t)+n(t) .
$$

The additive noise $n(t)$ is supposed to be stationary, white, and complex-Gaussian distributed. 


\section{ADAPTIVE EUCLIDEAN DistANCE MLSE RECEIVERS}

In this section, the MLSE Euclidean distance structure and the adaptive techniques needed for tracking the fast channel response variations, are described.

A new adaptive technique named "minimum-survivor" [16], [18], [19] is highlighted and compared with the per-survivor technique [7]. Among the adaptive realizations using the LMS and RLS methods, the different min-survivor and persurvivor estimation techniques have all exhibited very similar performance as shown in the simulation results. Consequently, the simplest structure which is the LMS min-survivor receiver, is the receiver proposed in the work.

The need to introduce the adaptive techniques within the Euclidean distance MLSE receiver arises since the likelihood branch function parameters and the correlator receiver depend on the time varying channel characteristics [17]. Only in the case of invariant time channel it is possible to measure its impulse response, allowing the receiver to be correctly initialized and permanently operate as a MLSE estimator. In the case of the land mobile radio link, the channel and the synchronization are always unknown and time-varying. Many land mobile communications systems employ the periodic transmission of some established short sequences (preamble) of symbols known by the receiver in order to give start-up values of the likelihood parameters (training processing). Furthermore, the parameters update can be subsequently carried out iteratively throughout the information sequence transmission by means of adaptive algorithms (tracking processing).

The employment of tracking techniques are justified by the following.

1) The training estimation is affected by noise and can be improved by further adaptive estimate.

2) The training estimation needs to be adjusted during the information bits according to the link parameter time changes imposed by fading [20], Doppler effects, and any incoherence phenomena.

The conventional adaptive MLSE receiver generally consists of an MLSE estimator implemented by the VA and a channel estimator which adaptively estimates the channel impulse response using the tentative decisions provided by VA as an estimate of the transmitted sequence [14], [15], [26]. The tentative decisions are obtained with a delay; if the delay is small, erroneous data decisions are given while a large delay causes a channel estimation delay decreasing the capability of tracking fast time-varying ISI channels [28]. Generally, the tentative decisions are made by truncating the survivor path history in the VA to some fixed length usually equal to $5 L$ ( $L$ indicates the memory length) giving a channel estimation delay. As suggested by Qureshi the channel estimation is obtained by using the tentative sequence with the largest survivor metric [5], [27].

In the literature, two similar methods, the per-survivor method [7] and the channel estimation procedure proposed by Kubo et al. [28], estimate the channel impulse response into the structure of the VA itself, along the survivor path connected to each state without the decision delay. In our method the channel estimation is made directly within the metric calculation of the VA with very restricted delay. The branch metric of the VA calculates the Euclidean distance between the received sequence and the estimate of received signal, therefore the VA directly provides the error signal used by the adaptive algorithms.

In the min-survivor processing, the sequence used in channel estimation corresponds to a particular state transition and is the sequence associated to the survivor path with the best metric value up to that time. However, one final decision is taken in favor of one sequence at the very end, once the entire transmitted signal has been received (at the end of the burst in the GSM) in order to minimize the error propagation.

The selected sequence is taken with a very restricted delay (the optimal value of the restricted delay is found by computer simulation) to avoid a degradation of tracking capability at each step of the VA.

According to the MLSE principle [3], the demodulation of digital information is accomplished through sequences, and a likelihood function is defined on the space of possible candidate sequences $\left\{\alpha_{n}\right\}$ which returns, for each sequence, a measure of its probability of transmission. This function depends on the system impulse response, on the power density spectrum of the additive noise introduced in the link, on the carrier phase and the symbol timing synchronization, as well as on the received signal.

To identify the ML sequence, it is possible to optimize the function $F\left(\left\{\alpha_{n}\right\}\right)$ with respect to the sequences using the VA, which is characterized by an only linear complexity (through a factor $2^{L-1}$ ), with the sequence length $N$ [13]. Each branch metric (or function), depends on $L$ consecutive bits in the sequence, where $L$ from the literature [4], [5] is equal to the received pulse duration $L_{S}$.

Therefore, in the presence of AWGN, the MLSE criterion leads to a receiver which searches among all possible data sequences to find the sequence closest to the noisy received signal, according to the Euclidean metric directly used in the VA [3].

The Euclidean distance MLSE branch function can be defined as follows:

$$
f_{n}=\left|y_{n}-\sum_{i=0}^{L-1} c_{n}(i) \alpha_{n-i}\right|^{2}
$$

where $y_{n}$ is the received signal and $c_{n}(i)$ are the branch function coefficients in which the introduction of dependence on time index $n$ accounts for their time changes.

The $c_{n}(i)$ can also be regarded as the coefficients of the linear finite impulse response (FIR) system that models the communication link.

The aim of adaptivity is to provide the best possible estimation of $c_{n}(i)$ parameters to the Viterbi processor. Since $c_{n}(i)$ are the coefficients of the time-varying FIR, tracking particularly consists of a certain procedure for the identification of an unknown time varying FIR by observing the flow of its input and output signals. One estimation problem occurs because while the output signal $y_{n}$ is available, the $\left\{a_{n}\right\}$ input sequence is unknown and has to be somehow approximated through information supplied by the Viterbi processor itself. 
Let $\mathbf{c}_{n}=\left[c_{n}(0), c_{n}(1), \cdots, c_{n}(L-1)\right]^{\prime}$ now be the vector of coefficients at time $n, \hat{\mathbf{c}}_{n}=\left[\hat{c}_{n}(0), \hat{c}_{n}(1), \cdots, \hat{c}_{n}(L-\right.$ 1)]' its estimation (' denotes transpose conjugate), $\boldsymbol{\alpha}_{n}=$ $\left[\alpha_{n}, \alpha_{n-1}, \cdots, \alpha_{n-L+1}\right]^{\prime}$ a possible transmitted $L$-uple, $\mathbf{a}_{n}=$ $\left[a_{n}, a_{n-1}, \cdots, a_{n-L+1}\right]^{\prime}$ the actually transmitted sequence. Most system identification methods are based on the criterion of the minimization of the mean square output error $E\left\{\left|e_{n}\left(\mathbf{a}_{n}, \hat{\mathbf{c}}_{n}\right)\right|^{2}\right\}$ with respect to $\hat{\mathbf{c}}_{n}[21]$, where

$$
e_{n}\left(\mathbf{a}_{n}, \hat{\mathbf{c}}_{n}\right)=y_{n}-\hat{\mathbf{c}}_{n}^{\prime} \mathbf{a}_{n}
$$

is the output error, depending on $\hat{\mathbf{c}}_{n}$. The branch function (11), generalized as a function of $\hat{\mathbf{c}}_{n}$ coefficients vector, takes the form

$$
f_{n}\left(\boldsymbol{\alpha}_{n}, \hat{\mathbf{c}}_{n}\right)=\left|y_{n}-\hat{\mathbf{c}}_{n}^{\prime} \boldsymbol{\alpha}_{n}\right|^{2}=\left|e_{n}\left(\boldsymbol{\alpha}_{n}, \hat{\mathbf{c}}_{n}\right)\right|^{2} .
$$

As a matter of fact, both the branch function and the mean square output error (to be minimized) are quadratic functions of the error. Using the Euclidean distance adaptive MLSE approach, some computational complexity can be avoided: the metric is computed by the Viterbi processor and is employed in the tracking algorithm as well.

A simple classical adaptive algorithm is the LMS [3], [6], [21], [22] which updates the coefficients in the direction of the $\left|e_{n}\left(\mathbf{a}_{n}, \hat{\mathbf{c}}_{n}\right)\right|^{2}$ gradient (versus $\mathbf{c}_{n}$ ). This results in the following iteration:

$$
\hat{\mathbf{c}}_{n+1}=\hat{\mathbf{c}}_{n}+\mu e_{n}^{*}\left(\mathbf{a}_{n}, \hat{\mathbf{c}}_{n}\right) \mathbf{a}_{n}
$$

where $\mu$ is the step-size parameter that controls the rate of adjustment. In theory, $\mu$ must be chosen as a compromise between the speed of response and the stability of the algorithm. In this paper, the optimization of $\mu$ has been performed by computer simulation of different system conditions.

Instead the RLS [3], [6], [21] algorithm attains the minimization of the function

$$
\sum_{i=0}^{n} w^{n-i}\left|e_{i}\left(\mathbf{a}_{i}, \hat{\mathbf{c}}_{n}\right)\right|^{2}=\sum_{i=0}^{n} w^{n-i}\left|y_{i}-\hat{\mathbf{c}}_{n}^{\prime} \mathbf{a}_{i}\right|^{2}
$$

where $w \in(0,1)$ is the "forgetting factor", weighing the past output errors according to an exponentially decreasing progression. In the time-invariant taps case, if the input is stationary, the RLS algorithm is asymptotically optimal for $w \simeq 1$ since it approximates the minimization of mean square output error law. Nevertheless, when the $c_{n}$ vector is timevarying, a forgetting factor must be introduced by reducing $w$. The coefficients computation can be efficiently performed iteratively as follows [3], [29]:

where

$$
\hat{\mathbf{c}}_{n+1}=\hat{\mathbf{c}}_{n}+e_{n}^{*}\left(\mathbf{a}_{n}, \hat{\mathbf{c}}_{n}\right) \mathbf{k}_{n}
$$

$$
\mathbf{k}_{n}=\frac{\mathbf{P}_{n-1} \mathbf{a}_{n}}{w+\mathbf{a}_{n}^{\prime} \mathbf{P}_{n-1} \mathbf{a}_{n}}
$$

is the Kalman weighing vector, and

$$
\mathbf{P}_{n}=\frac{1}{w}\left(\mathbf{P}_{n-1}-\mathbf{k}_{n} \mathbf{a}_{n}^{\prime} \mathbf{P}_{n-1}\right)
$$

is the input inverse autocorrelation matrix

$$
\mathbf{\Phi}_{n}=\sum_{i=0}^{n} w^{n-i} \mathbf{a}_{i} \mathbf{a}_{i}^{\prime} .
$$

Both the LMS and RLS algorithms update the $\hat{\mathbf{c}}_{n}$ by iteratively adding an adjustment term. The adjustment term is given by a vector of weights, which, multiplied by the error $e_{n}$, determines the parameter change. While the increase is forced in the $\mathbf{a}_{n}$ vector direction in the LMS algorithm and evaluates $\mu e_{n}$ (in absolute value) for each component, in the RLS algorithm the increase in one component is determined by the associated weight in the Kalman gain vector. The LMS and RLS algorithms update the channel coefficients within the VA and therefore the error $e_{n}$ (or the branch metric calculation) also depends on the state transitions, according to (13). The updating of the channel coefficients at time $(n+1)$ in the VA is based on the old least-squares estimate of the coefficients vector which was made at time $n$.

Since the sequence $\left\{a_{n}\right\}$ is not available, the LMS and RLS algorithms must use an estimate. To operate in realtime, the estimation of $\left\{a_{n}\right\}$ cannot be the tentative decisions supplied by the VA with fixed delay, but must be continuously updated from the information provided by the Viterbi processor at each step. In principle, the idea is to let the Viterbi processor operate in concurrence with the coefficients estimation processor. The Viterbi processor calculates the error, the metrics, the survivor paths, while the other processor updates the branch function coefficients. At each step $n$, the set of Viterbi survivor paths includes the path which will give the maximum likelihood sequence. Among them, that with the best metric value (minimum-survivor, briefly min-survivor) can be regarded as the most likely close to the signal samples received and processed up to that time.

The realization of minimum-survivor principle is quite simple since it only requires the comparison of all the survivor metrics at every step. The sequence with the largest metric is used to update the branch function coefficient to all the states of the VA. Let $\left\{\hat{a}_{0}(n), \hat{a}_{1}(n), \cdots, \hat{a}_{n}(n)\right\}$ be the min-survivor at time $n$, which is also the instantaneous maximum likelihood sequence at time $n$. It has been verified that the error rate in the above sequence is not uniform with respect to $n$, but can be significantly higher for values close to $n$ [23], [24]; in other words, the survivor paths converge in the past. Thus, in order to approximate the true transmitted sequence with the least errors, it is worthwhile to introduce some delay $d>0$ in the symbols $a_{n}$ of the recursions (14), (16)-(18). Of course, if the $d$ chosen is too large, the delay introduced in the estimation process can determine significant performance degradation.

An alternative to the above mentioned method is to accomplish an independent branch function coefficients estimation for each survivor (PPSP) [7]. In order to calculate the branch metric relative to a possible state transition, the coefficients associated to the source state are employed at each step. Then, after the new set of survivor paths is determined and the corresponding correct (surviving) state transitions are found, a coefficients vector updating is carried out for each state transition. The coefficients associated to the incoming state are 
updated in terms of: the coefficients associated to the source state; an adjustment increase depending on the error and the binary L-uple corresponding to the state transition.

Notice that the PPSP formally results in an extension of the VA. We find not only a metric for each survivor path, but a metric and other parameters (the branch function coefficients vector and, if RLS is used, the path inverse autocorrelation matrix which must be stored for each state to be updated iteratively). This feature makes the per-survivor approach attractive from a conceptual point of view although a theoretical comparison (in terms of performance) with the min-survivor approach is not easy to attain.

The LMS and RLS algorithms are used in the particular context of additive Gaussian-distributed noise, in which the squared output error is minimized. Therefore, if the true transmitted $L$-uple $\mathbf{a}_{n}$ is employed for coefficients updating at step $n$, since

$$
y_{n}=\mathbf{a}_{n}^{\prime} \mathbf{c}_{n}+n_{n}
$$

where $n_{n}$ are AWGN samples, the corresponding adjustment term can directly estimate the true coefficients vector $\mathbf{c}_{n}$. On the other hand, when a generic $L$-uple $\boldsymbol{\alpha}_{n}$ is employed, then the corresponding received signal model results in

$$
y_{n}=\boldsymbol{\alpha}_{n}^{\prime} \mathbf{c}_{n}+m_{n}
$$

where the additive noise term

$$
m_{n}=n_{n}+\left(\mathbf{a}_{n}-\boldsymbol{\alpha}_{n}\right)^{\prime} \mathbf{c}_{n}
$$

is no longer Gaussian-distributed, making the above mentioned algorithms unable to determine the adjustment term correctly at step $n$. Let us introduce the diagonal matrix $\mathbf{E}_{n}\left(\alpha_{n}\right)=$ $\operatorname{diag}\left\{\mathbf{a}_{n}\right\} \cdot \operatorname{diag}\left\{\boldsymbol{\alpha}_{n}\right\}$ whose generic element is: $e_{(i, j)}=0$ for $i \neq j(i, j=0, \cdots, L-1) ; e_{(i, i)}=+1$ if $\alpha_{n}(i)$ is "right" when compared to the corresponding true transmitted symbol $a_{n}(i)$ and $e_{(i, i)}=-1$ if it is "wrong" $(i=0, \cdots, L-1)$. It is easy to see that the signal model, if the $\boldsymbol{\alpha}_{n} L$-uple and AWGN is transmitted, can be written as

$$
y_{n}=\boldsymbol{\alpha}_{n}^{\prime} \mathbf{E}\left(\boldsymbol{\alpha}_{n}\right) \mathbf{c}_{n}+n_{n} .
$$

The adjustment term moves the estimation at step $n$ toward $\mathbf{E}_{n}\left(\boldsymbol{\alpha}_{n}\right) \mathbf{c}_{n}$ which disagrees with $\mathbf{c}_{n}$, as it has opposite components value in correspondence to wrong components in $\boldsymbol{\alpha}_{n}$. Now, when the per-survivor estimation is carried out at step $n$, since all the $L$-uple $\boldsymbol{\alpha}_{n}$ associated to the $2^{L-1}$ survivors differ from one another, it follows that at most one of the updating terms associated to each survivor is correct as well and the estimation moves in the right direction.

This statement does not imply that the per-survivor estimation paths are globally diverging. In fact at each step the global estimation history is similar for all the survivor paths, since all of them converge quickly back to the past [5]. Only in the steps preceding step $n$ the estimation paths tend to diverge. Particularly, the more errors appear inside a path in the most recent bits (particularly in the $L-1$ state bits), the more the final estimated coefficients associated to it may differ from the right ones. This is not, in general, a favorable condition for a
TABLE I

Numbers $N, N_{m}, N_{c}$, of Real Sums, Multiplications, Memory Cells Required Per Step by One Single Coefficients Vector Updating

\begin{tabular}{l|c|c|c}
\cline { 2 - 4 } & $\mathbf{N}_{\mathbf{s}}$ & $\mathbf{N}_{\mathrm{m}}$ & $\mathbf{N}_{\mathrm{c}}$ \\
\hline \hline $\mathbf{L M S}$ & $2 \mathbf{L}$ & 2 & $\mathbf{2 L}$ \\
\hline $\mathbf{R L S}$ & $2 \mathbf{L}^{2}+2 \mathbf{L}$ & $2 \mathbf{L}^{2}+3 \mathbf{L}$ & $\mathbf{L}^{2}+4 \mathbf{L}$ \\
\hline
\end{tabular}

correct demodulation, since some wrong survivor path could even worsen the bad estimation associated to them: e.g., the path corresponding to $\left\{-a_{n}\right\}$ sequence, if it is the survivor, could lead the coefficients estimation to its equilibrium point $-\mathbf{c}_{n}$ quite quickly and survive further. In addition, statistics estimated through computer simulations have shown that the survivor metrics exhibit smaller average and standard deviation values when the estimation technique is per-survivor instead of min-survivor. This condition may indicate a smaller ability of emphasizing the different likelihood paths by the per-survivor technique.

Finally, the computational complexity comparison for the two estimation techniques considered indicates that a significant amount of computation can be avoided using the min-survivor technique. The reason lies substantially in the fact that the min-survivor processing carries out one estimation process instead of $2^{L-1}$ (one per state) channel estimations needed for the PPSP. Particularly, the basic cost of a single vector of coefficient updating depends on the algorithm used (LMS or RLS) and can be expressed as the sum $N_{s} c_{s}+$ $N_{m} c_{m}+N_{c} c_{c}$ where $N_{s}, N_{m}, N_{c}$ are the real sums, real multiplications and memory cells required and $c_{s}, c_{m}, c_{c}$ are respectively the cost of one real sum, one multiplication and one memory cells. Table I lists the values of $N_{s}, N_{m}, N_{c}$ as a function of $L$. The computational complexity for the error $e_{n}$ is not included in Table I as this value is available by the Viterbi processor. The cost of min-survivor processing is then determined by the following additional quantities: the first, $c_{1}=\left(2^{L-1}-1\right) c_{s}+c_{c}$ is due to the minimum survivor search problem solution; the second, $c_{2}$ depends on the particular memory allocation of the survivor path (in any case linear with respect to $L$ parameter) and is due to the presence of delay $d$ in (14)-(18), which requires to look back in the minimum survivor path to find the bit vector $\hat{\mathbf{a}}_{n-d}(n)$ and evaluate the output error $e_{n-d}\left(\hat{\mathbf{a}}_{n-d}(n), \hat{c}_{n}\right)$. As a result, the estimation cost for min-survivor is

$$
c_{\text {min-surv }}=\left(N_{s} c_{s}+N_{m} c_{m}+N_{c} c_{c}\right)+c_{1}+c_{2}
$$

per step.

On the other hand the per-survivor computational complexity is $2^{L-1}$ times the basic cost, that is

$$
c_{\text {per-surv }}=2^{L-1}\left(N_{s} c_{s}+N_{m} c_{m}+N_{c} c_{c}\right)
$$

per step. Considering the values shown in Table I for $N_{s}, N_{m}, N_{c}$, the per-survivor/min-survivor computational complexities ratio grows with a power of $L$ in such a way that

$$
\lim _{L \rightarrow \infty} \frac{1}{L} \frac{c_{\text {per-surv }}(L)}{c_{\text {min-surv }}(L)}=2 c_{c} / c_{s}+2
$$


TABLE II

Channel Coefficients (Sampled Received Pulse)

\begin{tabular}{l||c|c}
\hline $\begin{array}{l}\text { channel } \\
\text { coeff. }\end{array}$ & real part & imag. part \\
\hline $\mathbf{c}(0)$ & 0.02 & 0.37 \\
\hline $\mathbf{c}(1)$ & 0.04 & -0.71 \\
\hline $\mathbf{c}(2)$ & -0.02 & 0.37 \\
\hline $\mathbf{c}(3)$ & 0 & -0.03 \\
\hline $\mathbf{c}(4)$ & 0 & 0 \\
\hline \hline
\end{tabular}

for LMS, and for RLS

$$
\lim _{L \rightarrow \infty} \frac{1}{L^{2}} \frac{c_{\text {per-surv }}(L)}{c_{\text {min-surv }}(L)}=2 c_{m} / c_{s}+c_{c} / c_{s}+2 .
$$

\section{Simulated Performance}

The performance of the different adaptive MLSE receivers has been evaluated experimentally according to the standard of the new high-capacity pan-European digital mobile radio system, the ETSI/GSM. This system [18] employs TDMA with 8 channels per carrier. All the carriers are located in the 900 $\mathrm{MHz}$ frequency band and are spaced by a $200 \mathrm{kHz}$ bandwidth. In the case of user data packet (normal burst), the information bits are split into two groups of 58 bits by a 26-bit long preamble sequence that enables the synchronization and the estimate of the channel response by a particular autocorrelation property. This autocorrelation [25] property optimizes training estimation of a system with no more than $L=5$ coefficients. The bit period evaluates $T=3.69 \mu \mathrm{s}$, hence the bit rate is $R=270.833 \mathrm{kbit} / \mathrm{s}$. The adopted modulation scheme is the GMSK signaling with normalized 3-dB bandwidth $\mathrm{BT}=0.3$ which is characterized by modulation index $h=1 / 2$, a smooth shaping pulse, narrow band, and belongs to the class of CPM described in Section II. Before entering the GMSK phase modulator, the binary sequence is differentially encoded as indicated in Section II, so that the linear model (7) can be used for the transmitted signal.

In order to assess performance and compare different MLSE receivers, various propagation models have been considered: AWGN channel with Doppler effect $(175 \mathrm{~Hz}$ carrier frequency constant shift corresponding to vehicle radial velocity 210 $\mathrm{km} / \mathrm{h}$ ); the fading multipath channels indicated as typical urban (TU), hilly terrain (HT) and rural area (RA) in GSM recommendations with mobile speed of respectively $50 \mathrm{~km} / \mathrm{h}$, $100 \mathrm{~km} / \mathrm{h}$ and $300 \mathrm{~km} / \mathrm{h}$, are simulated by the six specified taps. The employed transmitter and receiver bandpass filters have linear phase. Finally, the additive noise introduced by the channel simulator is white. The ratios of energy/b to white noise variance values $\left(E_{b} / N_{o}\right)$ of 3,5 , and $8 \mathrm{~dB}$ were simulated in the AWGN with Doppler channels, while 8, $16,24 \mathrm{~dB}$ were chosen for simulation in TU, HT, and RA.

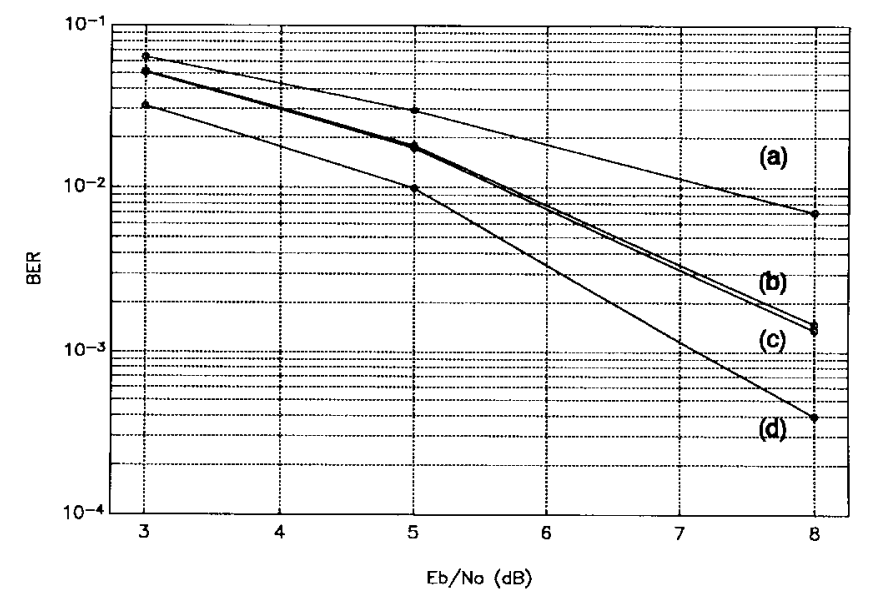

Fig. 1. AWGN channel with Doppler effect $f_{d}=175 \mathrm{~Hz}-$ MLSE BER performance: (a) nonadaptive, (b) LMS min-survivor, (c) LMS per-survivor, and (d) reference receiver.

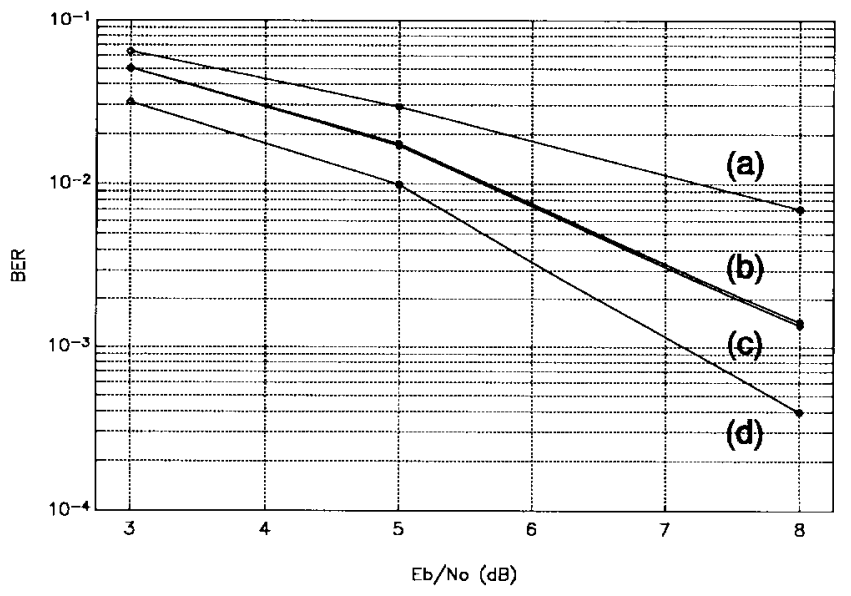

Fig. 2. AWGN channel with Doppler effect $f_{d}=175 \mathrm{~Hz}-$ MLSE BER performance: (a) nonadaptive, (b) RLS min-survivor, (c) RLS per-survivor, and (d) reference receiver.

The receiver sampling frequency has been chosen equal to bit rate, and the parameter $L$ has been regularly set to a value of 5 for the trials, so that the simulated demodulator is always a Viterbi of $2^{L-1}=16$ states. The values for the channel coefficients $c(i)$ (shown in Table II) are calculated using the true transmitted sequence and taking into account the modulation scheme, the AWGN channel, the transmitter and receiver filters.

The performance comparison among the "nonadaptive" receiver, the LMS/RLS per-survivor receiver and the LMS/RLS min-survivor receiver is evaluated in terms of the bit-error rate (BER) versus $E_{b} / N_{o}$. In the "nonadaptive" receiver, the known training sequence of each burst (midamble) is used to estimate the channel response during the actual burst. This training channel estimate is held fixed in the computing of the Viterbi metric for the entire burst duration and only the next burst is updated. The term "nonadaptive" is used in this section to indicate a periodic update of the channel parameters (at each burst). It differs from the adaptive (LMS/RLS min/persurvivor) receivers, which use this training channel estimate for start up purposes only and then update continuously the channel estimate within the burst. 


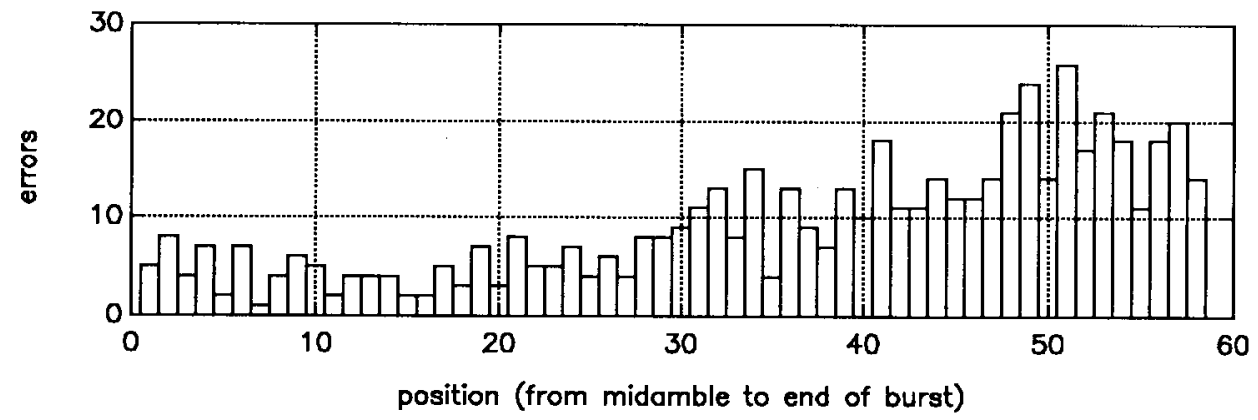

(a)

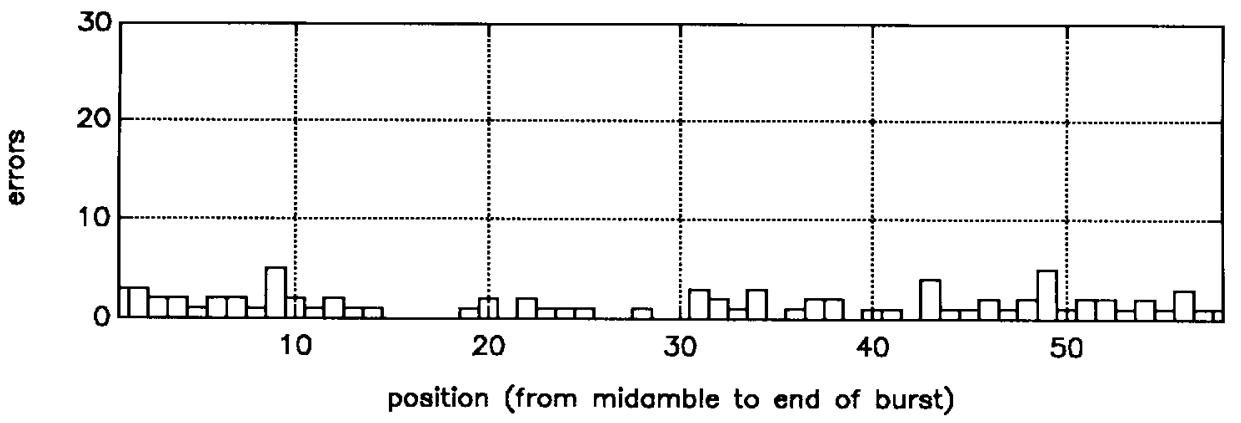

(b)

Fig. 3. AWGN channel with Doppler effect $f_{d}=175 \mathrm{~Hz}, E_{b} / N_{0}=5 \mathrm{~dB}$-hist. of errors $(1=$ midamble side, $58=$ end of burst side), stat. on 1000 burst: (a) nonadaptive and (b) RLS min-surv. $w=0.96$.

The performance of different receivers has been assessed by the demodulation of the same signal and noise realization, for each assigned channel type and $E_{b} / N_{o}$ ratio. The statistics extension is determined by the number of transmitted bursts simulated, which has been fixed on 1000 bursts. The results presented refer to optimized values of the estimation algorithms parameters. The optimization was carried out experimentally repeating the demodulation trials for different parameters values. For LMS algorithm, the optimal values of the step-size parameter $\mu$ have been found to range from 1/80 to $1 / 20$ in all cases. For RLS algorithm, the optimality for the forgetting factor $w$ is exhibited in the range $0.96 \div 0.99$. When min-survivor estimation technique was employed, the delay parameter $d$ had been optimized and the values chosen were $d=1 T$ ( $T$ is the bit period) for AWGN+Doppler, RA channels and $d=2 T$ for TU, HT channel.

\section{A. Results}

1) AWGN Channel with Doppler Effect: These simulations refer to an impairment introduced by multiplying the transmitted signal (its complex envelope) by the exponential $e^{j 2 \pi f d t}$, with $f_{d}=175 \mathrm{~Hz}$ (corresponding to $210 \mathrm{~km} / \mathrm{h}$ vehicle speed, which is a $16^{\circ}$ phase shift in half burst), in order to describe the progressive rotation of received pulse due to the presence of Doppler effects. The results are shown in Figs. 1-2. The reference curve is associated to the receiver that permanently uses the perfect AWGN channel coefficients (Table II).

The adaptive techniques allow an effective tracking of parameters. A $2 \mathrm{~dB}$ improvement is attained with respect to nonadaptive receiver at $\mathrm{BER}=2 \times 10^{-3}$.
This result is confirmed by the histogram depicted in Fig. 3: while the adaptive receivers keep the BER constant (the tracking processing already begins in the midamble sequence), the nonadaptive demodulation errors grow from midamble to the end of burst.

2) GSM RA Channel: In this environment the vehicle speed is equal to $300 \mathrm{~km} / \mathrm{h}$. The Doppler effects due to the high vehicle speed and the fading contribute to the degradation of the transmitted signal. The multipath effect does not change the channel response length significantly since the maximum simulated delayed path arrives to the receiver antenna 1/7T later than the direct path. However, the multipath degrades the BER of the receiver. The simulation results are presented in Figs. 4-5. A performance improvement of $5 \mathrm{~dB}$ at $\mathrm{BER}=10^{-2}$ is exhibited for adaptive receivers versus nonadaptive ones.

3) GSM TU Channel: This environment is characterized by moderated Doppler effects (simulated vehicle speed: 50 $\mathrm{km} / \mathrm{h}$ ), and multipath effect (1.3 $T$ maximum simulated delay) due to the presence of significant reflectors, such as large building walls. Figs. 6-7 depict the performance comparison respectively for LMS and RLS adaptive receiver with nonadaptive receiver. The improvement for adaptive receivers reaches $3 \mathrm{~dB}$ at $\mathrm{BER}=4 \times 10^{-4}$. Once again, no relevant differences are observed between LMS/RLS algorithm and $\mathrm{min} /$ per-survivor techniques.

4) GSM HT Channel: In this model the vehicle speed is equal to $100 \mathrm{~km} / \mathrm{h}$. Due to large time delays determined in some paths by the signal reflection on the mountain surfaces according to the GSM specifications, a relevant amount of energy arrives to the receiver antenna even $8 T$ later than the 


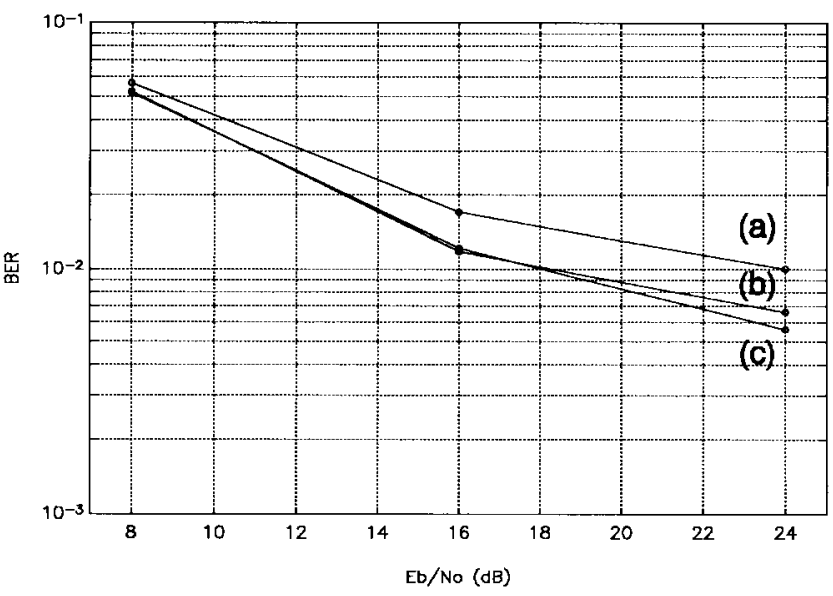

Fig. 4. GSM RA channel-MLSE BER performance: (a) nonadaptive, (b) LMS min-survivor, and (c) LMS per-survivor.

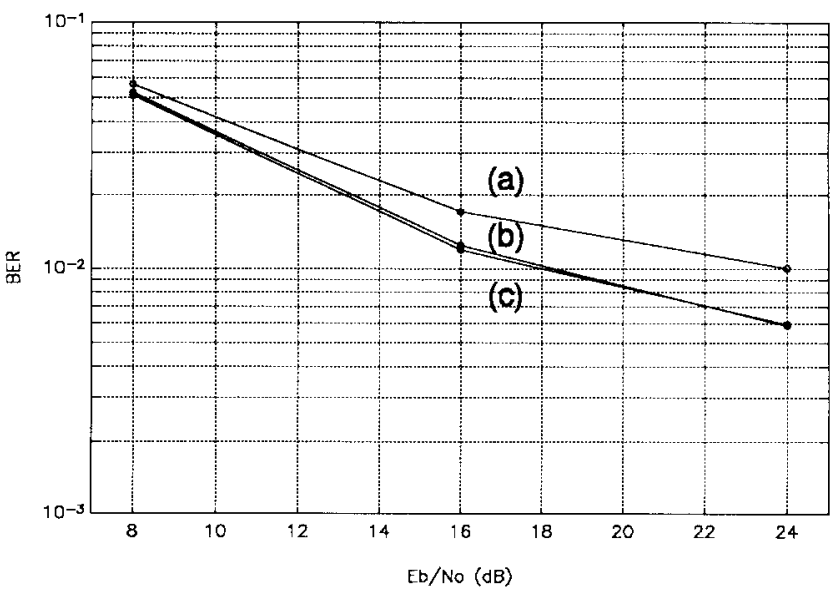

Fig. 5. GSM RA channel-MLSE BER performance: (a) nonadaptive, (b) RLS min-survivor, and (c) RLS per-survivor.

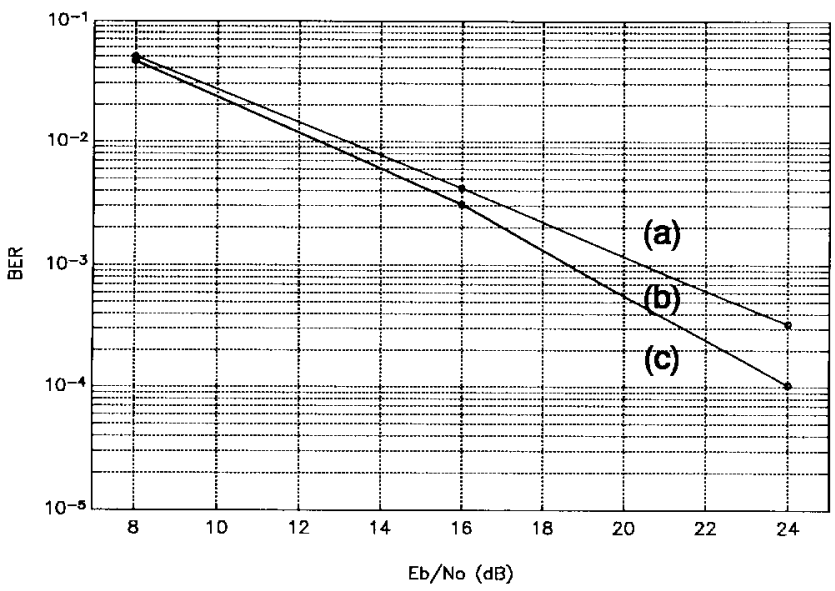

Fig. 6. GSM TU channel-MLSE BER performance: (a) nonadaptive, (b) LMS min-survivor, and (c) LMS per-survivor.

direct path. This energy results in additive noise since the length of the adopted Viterbi memory $(L=5)$ is not sufficient to recover such a large multipath effect. Moreover, in using a Viterbi receiver with memory $L$ equal to five, no improvement is obtained with adaptive techniques as shown in Figs. 8-9.

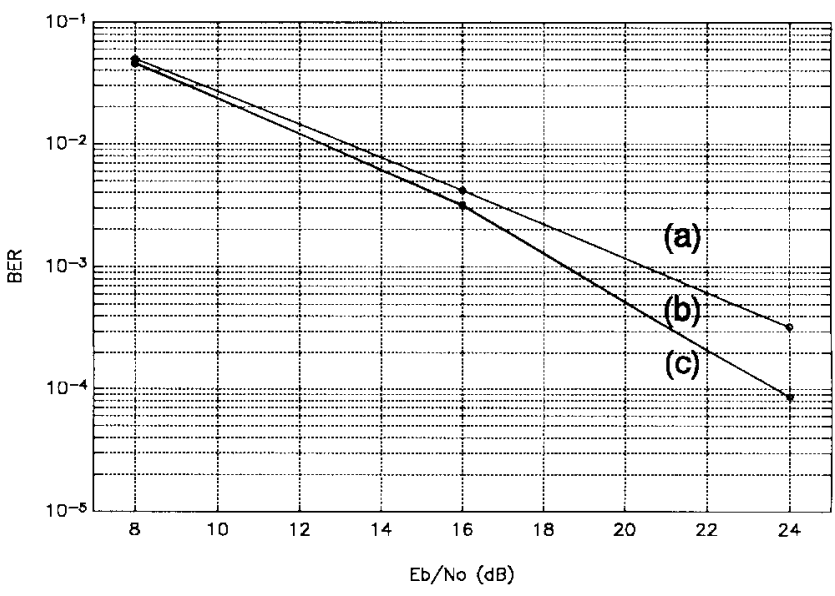

Fig. 7. GSM TU channel-MLSE BER performance: (a) nonadaptive, (b) RLS min-survivor, and (c) RLS per-survivor.

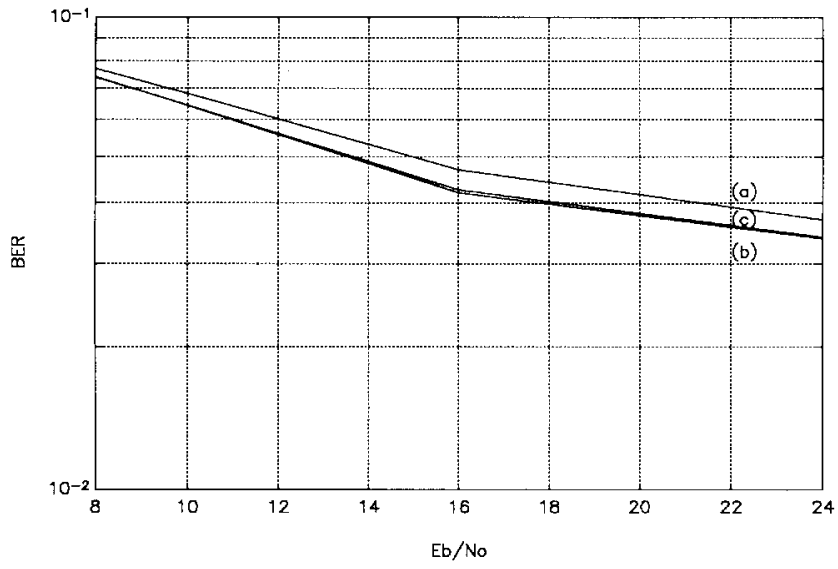

Fig. 8. GSM HT channel-MLSE BER performance: (a) nonadaptive, (b) LMS min-survivor, and (c) LMS per-survivor.

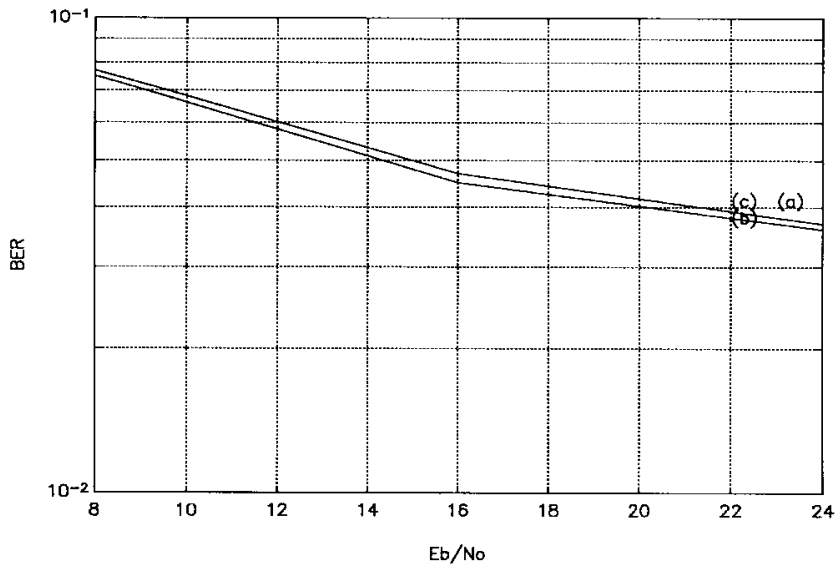

Fig. 9. GSM HT channel-MLSE BER performance: (a) nonadaptive, (b) RLS min-survivor, (c) RLS per-survivor.

\section{CONCLUSION}

Topics about a digital Euclidean distance MLSE structure have been discussed in the paper and data-aided adaptive realizations of the Euclidean distance MLSE receiver have been defined. Their performance has been studied in particular 
for the ETSI/GSM TDMA format in AWGN, Doppler and fading channel environments. Among the adaptive achievements, the different LMS/RLS per-survivor and LMS/RLS min-survivor estimation techniques have all exhibited very similar performance. Consequently, the simplest structure, which is the proposed LMS min-survivor receiver, can be regarded with particular interest.

\section{REFERENCES}

[1] C.-E. Sundberg, "Continuous phase modulation," IEEE Commun. Mag., vol. 24, no. 4, pp. 25-38, Apr. 1986.

[2] P. A. Laurent, "Exact and approximate construction of digital phase modulation by superimposition of amplitude modulated pulses (AMP)," IEEE Trans. Commun., vol. COM-34, no. 2, Feb. 1986.

[3] J. G. Proakis, Digital Communications. New York: McGraw-Hill, 1989.

[4] G. D. Forney, "Maximum-likelihood sequence estimation of digital sequences in the presence of intersymbol interference," IEEE Trans. Inform. Theory, vol. IT-18, no. 3, May 1972.

[5] G. Ungerboeck, "Adaptive maximum-likelihood receiver for carriermodulated data transmission systems," IEEE Trans. Commun., vol. 22, no. 5, May 1974.

[6] J. G. Proakis, "Adaptive equalization for TDMA digital mobile radio," IEEE Trans. Veh. Technol., vol. 40, no. 2, May 1991.

[7] R. Raheli, A. Polydoros, and C.-K. Tzou, "The principle of per-survivor processing: A general approach to approximate and adaptive MLSE," in Proc. GLOBECOM'91, Phoenix (USA), Dec. 1991.

[8] K. Murota and K. Hirade, "GMSK modulation for digital mobile telephony," IEEE Trans. Commun., vol. 29, no. 7, July 1981.

[9] A. Baier, "Derotation techniques in receivers for MSK-type CPM signals," Signal Processing V: Theories and Application. Elsevier Science Publishers, 1990.

[10] W. R. Braun and U. Dersch, "A physical mobile radio channel model," IEEE Trans. Veh. Technol., vol. 40, no. 2, May 1991

[11] G. A. Arredondo, W. H. Chriss, and E. H. Walker, "A multipath fading simulator for mobile radio," IEEE Trans. Commun., vol. COM-21, no. 11, Nov. 1973.

[12] GSM recommendation 05.05 (version 3.7.0).

[13] D. Messerschmitt and E. Lee, Digital Communications. Boston, MA: Kluwer, 1988.

[14] R. D'avella, L. Moreno, and M. Sant'Agostino, "An adaptive MLSE receiver for TDMA digital mobile radio," IEEE J. Select. Areas Commun., vol. 7, no. 1, Jan. 1989.

[15] K. Fukawa and H. Suzuki, "Adaptive equalization with RLS-MLSE for frequency-selective fast fading mobile radio channels," in Proc. GLOBECOM'91, Phoenix (USA), Dec. 1991.

[16] F. Conti, "Ricevitori digitali MLSE adattivi per sistemi radio mobili," Graduation dissertation, University of Florence, Florence, Italy, 1992.

[17] E. Del Re, "Adaptive channel estimation for mobile radio," in Proc. COST 229 Workshop on adaptive Algorithms: Application and Non Classical Schemes, Vigo Spain, Mar. 1991.

[18] E. Del Re, G. Castellini, L. Pierucci, and F. Conti, "A within burst adaptive MLSE receiver for mobile TDMA cellular systems," in Proc. IEEE ICASSP'92, San Francisco, CA, Mar. 1992

[19] _ _An improved adaptive MLSE receiver for fading communications channels," in Proc. EURASIP EUSIPCO'92, Brussells, Aug. 1992.

[20] R. A. Ziegler and J. M. Cioffi, "Estimation of timevarying digital mobile radio channels," in Proc. GLOBECOM'91, Phoenix (USA), Dec. 1991.

[21] B. Widrow and S. D. Stearns, Adaptive Signal Processing. Englewood Cliffs, NJ: Prentice-Hall, 1985.

[22] J. R. Zeidler, "Performance analysis of LMS adaptive prediction filters," Proc. IEEE, vol. 78, no. 12, Dec. 1990.

[23] G. D. Forney, "Review of random tree codes," NASA Amer. Res. Cen., Moffett Field, CA, Contract NAS2-3637, NASA CR 73176, Final Rep., Dec. 1967.

[24] A. J. Viterbi, "Convolutional codes and their performance in communication systems," IEEE Trans. Commun. Technol., vol. COM-19, Oct. 1971.

[25] S. N. Crozier, D. D. Falconer, and S. A. Mahmoud, "Least sum of squared errors (LSSE) channel estimation," in IEE Proc.-F, vol. 138, no. 4, Aug. 1991.

[26] J. H. Lodge and M. L. Moher, "Maximum likelihood sequence estimation of CPM signals transmitted over Rayleigh flat-fading channels," IEEE Trans. Commun., vol. 38, no. 6, June 1990.
[27] N. Seshadri, "Joint data detection and channel estimation using fast blind trellis search techniques," in Proc. GLOBECOM'90 Conf., Dec. 1990, pp. 1659-1663.

[28] H. Kubo, K. Murakami, and T. Fujino, "An adaptive maximum likelihood sequence estimator for fast time-varying intersymbol interference channels," IEEE Trans. Commun., vol. 42, no. 2/3/4, Feb./Mar./Apr. 1994.

[29] S. Haykin, Adaptive Filter Theory. Englewood Cliffs, NJ: PrenticeHall, 1991.

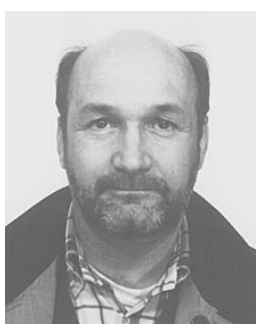

Guido Castellini was born in Firenze, Italy in 1944. $\mathrm{He}$ received the Laurea degree in physics from the University of Firenze, Italy, in 1968.

In 1971, he joined the Institute of Research on Electromagnetic Waves (IROE) of the Italian National Research Council, where he holds the position of Senior Researcher. His main current interests are in electronic system design for high energy physics experiments, in digital signal processing, and in neural processing applied to problems of nonlinear estimation.

Mr. Castellini is an Associated Member of the Italian National Institute for Nuclear Physics (INFN).

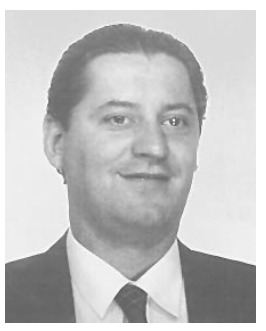

Fabrizio Conti was born in Florence, Italy, in 1965. He received the Dr. Ing. degree in electronic engineering from the University of Florence, Italy, in 1992.

Until 1993, he worked at the Department of Electronics Engineering of the University of Florence on research programs about MLSE receivers and adaptive filtering for digital radio mobile communications. Since 1993, he has been engaged in the Technical Staff as Software Engineer at Alcatel Italia Inc. His interest comprises radio mobile communications, digital demodulation techniques, object oriented programming languages, database management systems, and computer network communications.

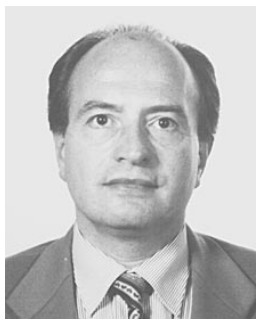

Enrico Del Re (M'78-SM'84) was born in Florence, Italy, in 1947. He received the Dr. Ing. degree in electronics engineering from the University of Pisa, Italy, in 1971.

Until 1975, he was engaged in public administration and private firms, involved in the analysis and design of the telecommunication and air traffic control equipment and space systems. Since 1975, he has been with the Department of Electronics Engineering of the University of Florence, Florence, Italy, first as a Research Assistant, then as an Associate Professor, and since 1986 as Professor. During the academic year 1987-1988, he was on leave from the University of Florence for a nine-month period of research at the European Space Research and Technology Centre of the European Space Agency, The Netherlands. His main research interest are digital signal processing, digital transmission techniques, and communication networks, on which he has published more than 150 papers, in international journals and conferences. He is the Co-Editor of the book Satellite Integrated Communications Networks (North-Holland, 1988), one of the authors of the book Data Compression and Error Control Techniques with Applications (New York: Academic, 1985) and the editor of the book Mobile and Personal Communications (Amsterdam, The Netherlands: Elsevier, 1995).

Dr. Del Re received the 1988/89 premium from the IEE (UK) for the paper "Multicarrier demodulator for digital satellite communication systems." He has been the Chairman of the European Project COST 227 "Integrated Space/Terrestrial Mobile Networks." He is a member of the AEI, the European Association for Signal Processing (EURASIP) and has been included in the edition of Who's Who in the World. 


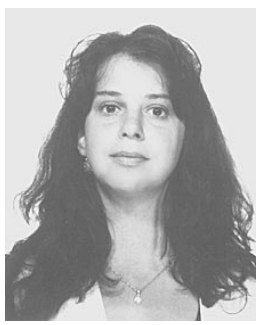

Laura Pierucci was born in Pistoia, Italy, in 1959.

She received the Dr. Ing. degree in electronics engineering from the University of Florence, Italy, in 1987.

Until 1991, she was a Research Consultant for private firms in the fields of biomedical ultrasonic applications and of the development of radio mobile cellular system. Since 1991, she has been with the Department of Electronic Engineering of the University of Florence as Technical Assistant. Her main research interests are digital signal processing, adaptive systems, neutral network, and mobile communications packet radio systems. Currently, she is participating in the European ISIS project on interactive satellite multimedia information system.

Dr. Pierucci received the Fellowship of the Italian Research National Council on optical multiplexing techniques in data transmission networks in 1989. 